EPJ Web of Conferences 40, 06011 (2013)

DOI: $10.1051 /$ epjconf/20134006011

(C) Owned by the authors, published by EDP Sciences, 2013

\title{
Magnetic flux concentration methods for magnetic energy harvesting module
}

\author{
Kunihisa Tashiro ${ }^{1}$, Gen-ya Hattori ${ }^{1}$, and Hiroyuki Wakiwaka ${ }^{1}$ \\ ${ }^{1}$ Spin Device Technology Center, Shinshu University, Nagano, Japan
}

\begin{abstract}
This paper presents magnetic flux concentration methods for magnetic energy harvesting module. The purpose of this study is to harvest $1 \mathrm{~mW}$ energy with a Brooks coil $2 \mathrm{~cm}$ in diameter from environmental magnetic field at $60 \mathrm{~Hz}$. Because the harvesting power is proportional to the square of the magnetic flux density, we consider the use of a magnetic flux concentration coil and a magnetic core. The magnetic flux concentration coil consists of an air-core Brooks coil and a resonant capacitor. When a uniform magnetic field crossed the coil, the magnetic flux distribution around the coil was changed. It is found that the magnetic field in an area is concentrated larger than 20 times compared with the uniform magnetic field. Compared with the air-core coil, our designed magnetic core makes the harvested energy ten-fold. According to ICNIRP2010 guideline, the acceptable level of magnetic field is $0.2 \mathrm{mT}$ in the frequency range between $25 \mathrm{~Hz}$ and $400 \mathrm{~Hz}$. Without the two magnetic flux concentration methods, the corresponding energy is limited to $1 \mu \mathrm{W}$. In contrast, our experimental results successfully demonstrate energy harvesting of $1 \mathrm{~mW}$ from a magnetic field of 0.03 $\mathrm{mT}$ at $60 \mathrm{~Hz}$.
\end{abstract}

\section{Introduction}

We have proposed magnetic energy harvesting from our environmental magnetic field [1-3]. Because power-lines are necessary for our modern life, we are required to coexist with undesirable magnetic power-line noise. ICNIRP2010 provides a guideline that an acceptable level for human health in a public space is $0.2 \mathrm{mT}$ in the power-line frequency range between $25 \mathrm{~Hz}$ and $400 \mathrm{~Hz}$ [4]. From a view point of researcher related with magnetic engineering, we focus on the field as a source of energy harvesting.

In previous reports, our developed modules demonstrated magnetic energy harvesting of $6.32 \mathrm{~mW}$ [1] and $104 \mathrm{~mW}$ [3] from $60 \mathrm{~Hz}$ magnetic field of $21.2 \mu \mathrm{T}$ and $90 \mu \mathrm{T}$, respectively. Because an ultra-low power wireless sensor node can be activated with $1 \mathrm{~mW}$ energy, it is possible to construct a wireless sensor network without a battery [5].

In general, solar power is a promising power source for energy harvesting. The power density of direct sunlight is about $1,000 \mathrm{~W} / \mathrm{m}^{2}$, and solar panels could harvest $10 \%$ of this energy [6]. Although magnetic energy is not affected by weather conditions, the reusable energy is defined by several guidelines. For example, the corresponding power density given by ICNIRP2010 is 10 $\mathrm{W} / \mathrm{m}^{2}$ in the microwave frequency range [4]. Because magnetic harvesting power is proportional to the square of the magnetic flux density, we focus on the magnetic flux concentration methods.

The purpose of this study is to harvest $1 \mathrm{~mW}$ of energy with a Brooks coil $2 \mathrm{~cm}$ in diameter from the environmental magnetic field at $60 \mathrm{~Hz}$. It is a challenge because the estimated energy with this air-core coil from a magnetic field of $0.2 \mathrm{mT}$ at $60 \mathrm{~Hz}$ is $1 \mu \mathrm{W}$. We have already reported the magnetic flux distribution around an air-core Brooks coil with a resonant capacitor is changed [2]. The first method is the use of the coil as a magnetic flux concentration coil. It can provide an area whose magnetic field is 20 times larger when compared with the applied uniform magnetic field. The second method is the use of a dumbbell-shaped magnetic core. In previous report [1], rod-shaped magnetic cores did not dramatically enhance the harvested energy. In contrast, the proposed core achieves the harvested energy ten-fold. By incorporating both methods, we have successfully demonstrated energy harvesting of $1 \mathrm{~mW}$ from a magnetic field of $0.03 \mathrm{mT}$ at $60 \mathrm{~Hz}$.

\section{Theoretical estimation}

Fig.1(a) shows the equivalent circuit of the energy harvesting module. Here, $L(\mathrm{H})$ is coil inductance, $R(\Omega)$ is coil resistance, $C(\mathrm{~F})$ is resonance capacitor and $R_{\mathrm{L}}(\Omega)$ is dummy load. If the dummy load is none, the magnetic flux concentration coil could be described the same

This is an Open Access article distributed under the terms of the Creative Commons Attribution License 2.0, which permits unrestricted use, distribution, and reproduction in any medium, provided the original work is properly cited. 
circuit. From Faraday's law of induction and Thevenin's theorem, the amplitude of the voltage source $V_{\text {in }}(\mathrm{V})$ can be expressed by

$$
V_{\text {in }}=2 \pi^{2} f n a^{2} \times\left(\mu_{0} H\right)[\mathrm{V}] \text {. }
$$

Where $f(\mathrm{~Hz})$ is the frequency of magnetic field, $n$ (turn) is the number of coil windings, $a(\mathrm{~m})$ is mean radius of coil and $\mu_{0} H(\mathrm{~T})$ is the mean flux density crossed with the mean cross section of the air-core coil. From the maximum power transfer theorem, we chose a load $R_{\mathrm{L}}$ $(\Omega)$ to the same as the coil resistance and used the voltage drop as the output voltage $V_{\text {out }}(\mathrm{V})$ for calculating the harvesting power $W(\mathrm{~W})$ with

$W=V_{\text {out }}^{2} / R_{\mathrm{L}}=V_{\text {in }}^{2} /(4 R)[\mathrm{W}]$.

Substituting equation (2) gives

$W=\left(\pi^{4} f^{2} n^{2} a^{4} / R\right) \times\left(\mu_{0} H\right)^{2}[\mathrm{~W}]$.

This means that the harvesting power is proportional to the square of the magnetic flux density.

Fig. 1(b) shows the concept of the magnetic flux concentration methods. When an environmental magnetic field crosses to magnetic concentration coil, consists of a relatively large air-core coil and resonant capacitor, a current is induced in the coil. The induced current produces a magnetic field around the coil. At the resonance frequency of the coil, the amplitude and direction of this magnetic field are relatively large and antiparallel to the environmental magnetic field. Here, we define the amplitude ratio of the synthesis magnetic field to environmental magnetic field as $g$, magnetic flux concentration ratio. If the energy harvesting module is placed at the magnetic concentration area, the following equation can be obtained.

$$
\begin{aligned}
& \alpha W=\left(\pi^{4} f^{2} n^{2} a^{4} / R\right) \times\left(\mu_{\mathrm{eff}} \times g \times \mu_{0} H\right)^{2}[\mathrm{~W}] \\
& \alpha=\left(\mu_{\mathrm{eff}} \times g\right)^{2}
\end{aligned}
$$

Where $\mu_{0} H$ represents mean environmental magnetic field, $\mu_{\text {eff }}$ represents the effective permeability of a magnetic core in the energy harvesting module, $\alpha$ represents the energy enhance ratio which is proportional to the square of the product of $g$ and $\mu_{\text {eff. The purpose of }}$ this study is to harvest $1 \mathrm{~mW}$ energy with a Brooks coil of $2 \mathrm{~cm}$ in diameter. Because the estimated harvesting power is $1 \mu \mathrm{W}$ from a magnetic field of $0.2 \mathrm{mT}$ at $60 \mathrm{~Hz}$, the required value of $\alpha$ is larger than 1,000 .

\section{Experimental Setup}

\subsection{Magnetic flux concentration coil}

We chose the shape of both coils, the energy harvesting module and magnetic flux concentration coil, is Brooks coil. Table 1 shows the specifications for the module and the concentration coil. A Brooks coil is a circular coil having a square cross section, and the ratio of the coil length, inner diameter, and outer diameter is 1:2:4. In order to estimate the inductance, the required number of parameter is only two, and the estimation error due to the spacing factor and tolerance is quite low [7]. In a previous report [2], we have investigated the effect of distance between arrayed modules on energy harvesting ability. It was found that the measured magnetic flux distribution around an air-core Brooks coil affects the harvested energy. In this paper, we focus on the magnetic flux distribution near the air-core part. Fig. 2 shows the experimental setup. In order to demonstrate an environmental magnetic field, we used Simple-Cubic-3 coil system (SC3) whose side length was $2 \mathrm{~m}$ [8].

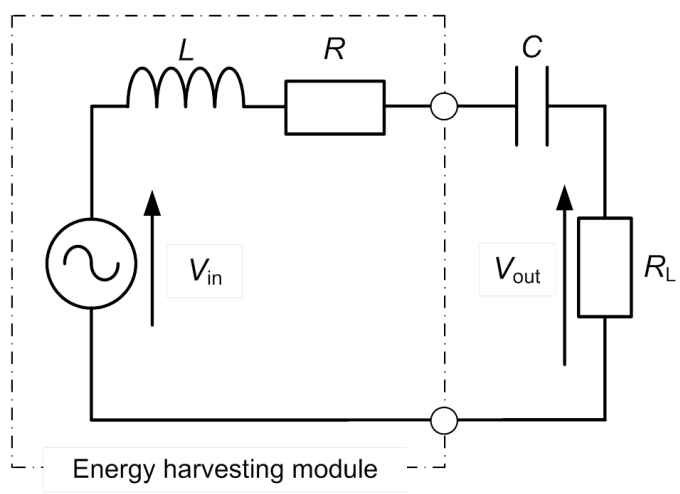

(a) Equivalent circuit of energy harvesting module

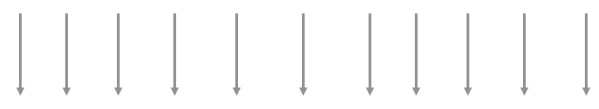

Magnetic flux concentration coil

(1) $\mu_{0} H$

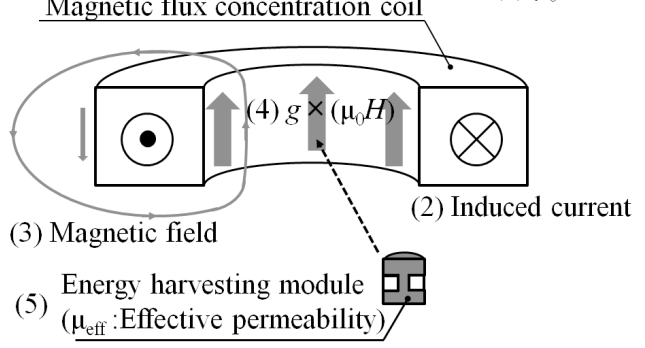

(b) Concept

Fig. 1. Concept and equivalent circuit of energy harvesting module.

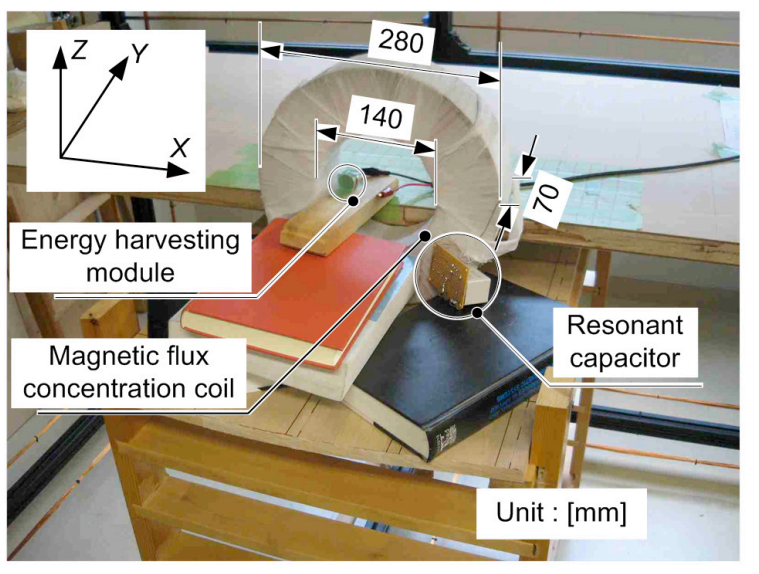

Fig. 2. Experimental setup. 
Table 1. Specifications for the harvesting module and magnetic flux concentration coil.

\begin{tabular}{l|cc}
\hline \multirow{2}{*}{ Property } & \multicolumn{2}{|c}{ Value } \\
\cline { 2 - 3 } & $\begin{array}{c}\text { Harvesting } \\
\text { module }\end{array}$ & $\begin{array}{c}\text { Concentration } \\
\text { coil }\end{array}$ \\
\hline Outer diameter, $D_{\mathrm{o}}[\mathrm{mm}]$ & 20 & 280 \\
\hline Number of windings, $n$ & 500 & 18395 \\
\hline Resonant capacitor, $C[\mu \mathrm{F}]$ & - & 0.1 \\
\hline Inductance, $L[\mathrm{mH}]$ & & \\
Estimated (Air core) & 3.19 & $60.6 \times 10^{3}$ \\
Measured (Air core) & 3.19 & $60.4 \times 10^{3}$ \\
(Magnetic core)Length: $7 \mathrm{~mm}$ & 21.06 & - \\
(Magnetic core)Length: $45 \mathrm{~mm}$ & 39.48 & - \\
\hline Resistance, $R[\Omega]$ & & \\
Estimated (Air core) & 13.35 & 1100 \\
Measured (Air core) & 1480 & 1060 \\
(Magnetic core)Length:7 mm & 14.27 & - \\
(Magnetic core)Length: $45 \mathrm{~mm}$ & 14.36 & - \\
\hline
\end{tabular}

We measured the flux distribution in the axial direction when a uniform magnetic field of $60 \mathrm{~Hz}$ crosses to the magnetic flux concentration coil, and calculated the magnetic concentration ratio. For this measurement, we used an induction magnetometer (3470, HIOKI).

\subsection{Magnetic core}

In previous report, we have only focused on the "equivalent" effective permeability inside the rod-shaped core [1]. However, the magnetic flux density is not uniform inside the rod-shaped core. In this paper, we focused on the effective permeability, or apparent permeability [9], defined by the enhance ratio of flux linkage to the coil with a magnetic material. Because a rod-shaped material has the maximum flux density at the center position, we make a constriction part in the area for winding the Brooks coil. The importance of the constriction part, or disk shaped flux concentrator [9], has been pointed out by some researchers. Fig. 3 shows magnetic cores we developed, and Table 1 shows the specifications. The diameter is $20 \mathrm{~mm}$, the material is PC Permalloy. We prepared five cores whose lengths are 7, 9, 15,25 , and $45 \mathrm{~mm}$, respectively. All cores have a constriction part whose diameter and length are $10 \mathrm{~mm}$ and $5 \mathrm{~mm}$, respectively. Because the own cutoff frequency of the coil is higher than $60 \mathrm{~Hz}$, a resonant capacitor is not connected.

\section{Results}

\subsection{Magnetic flux concentration coil}

Fig. 4 shows an experimental result of measured magnetic field distribution around the flux concentration coil. The applied magnetic field was $1 \mu \mathrm{T}$ at $60 \mathrm{~Hz}$. It was found that the measured magnetic field in the aircore area was larger than 10 times compared with the applied field. The maximum magnetic flux density was about $20 \mu \mathrm{T}$ which corresponds to the magnetic concentration ratio $g$ of 20 . Although we checked the dependency of the applied field strength on the magnetic concentration ratio, there was no significant difference when the amplitude was $10 \mu \mathrm{T}$. It means that we can enhance the usable magnetic energy in the air-core area up to 400 times.

\subsection{Magnetic core}

Fig. 5 shows the harvested power with harvesting modules as a function of applied magnetic field, as a parameter of the length of the magnetic core. In this experiment, the magnetic flux concentration coil was not used. The plots represent the measured values, and the line represents the estimated value when no magnetic core was used. The estimated values of effective permeability were also noted in this figure.

It was found that an increase in the length of the core produced an increase in the harvested energy. And the harvested energy was proportional to the square of the applied field strength. It means that the nonlinearity of the magnetic material was not found in this applied field strength range. When the length of the core was $45 \mathrm{~mm}$, the energy enhance ratio was 160 which corresponds to the effective permeability of 12.63 . In previous report [1], the maximum energy enhance ratio was 1.27 with relatively long magnetic rods. It should be noted that the energy enhance ratio was larger than 10 if the length of the core was $7 \mathrm{~mm}$. We concluded that the flange part of the magnetic core is important to concentrate the magnetic flux to the coil [9].

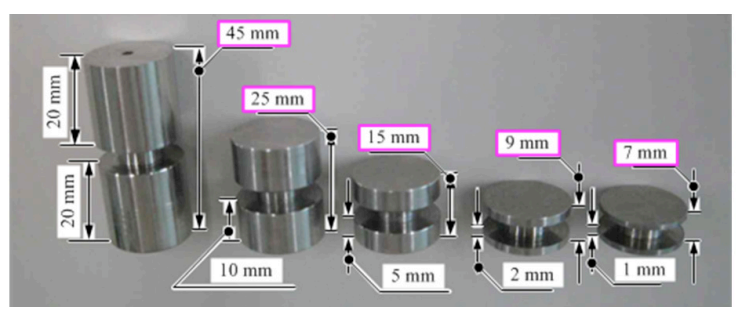

Fig. 3. Magnetic cores for the energy harvesting modules.

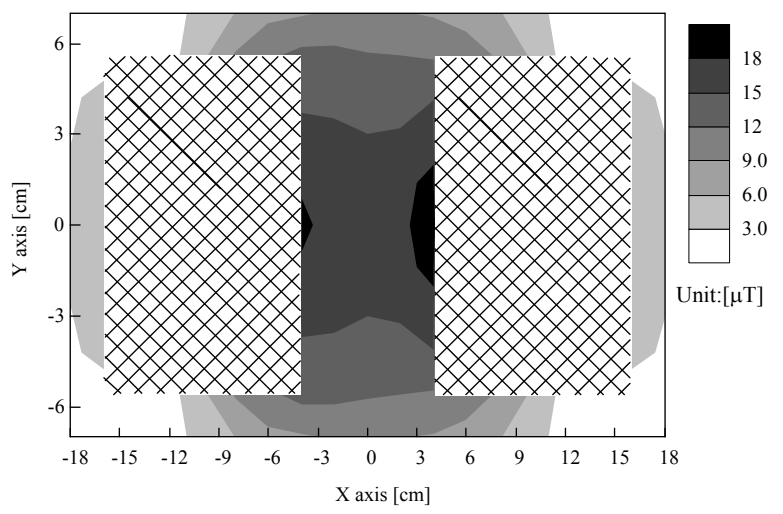

Fig. 4. Magnetic field distribution around the magnetic flux concentration coil. The direction of applied and calculated magnetic field was $\mathrm{Y}$ (axial) direction, and the applied uniform magnetic field was $1 \mu \mathrm{T}$ at $60 \mathrm{~Hz}$. 


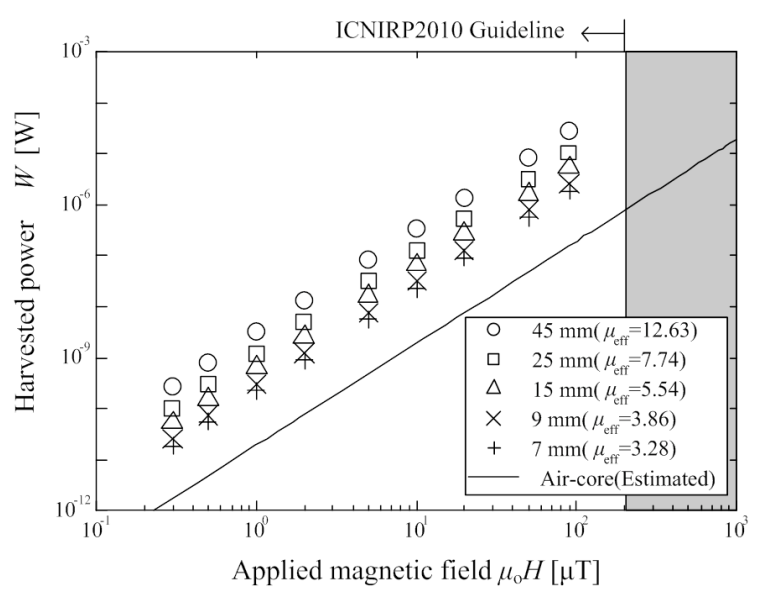

Fig. 5. Harvested power of the energy harvesting modules as a function of applied magnetic field, as a parameter of the length of the magnetic core.

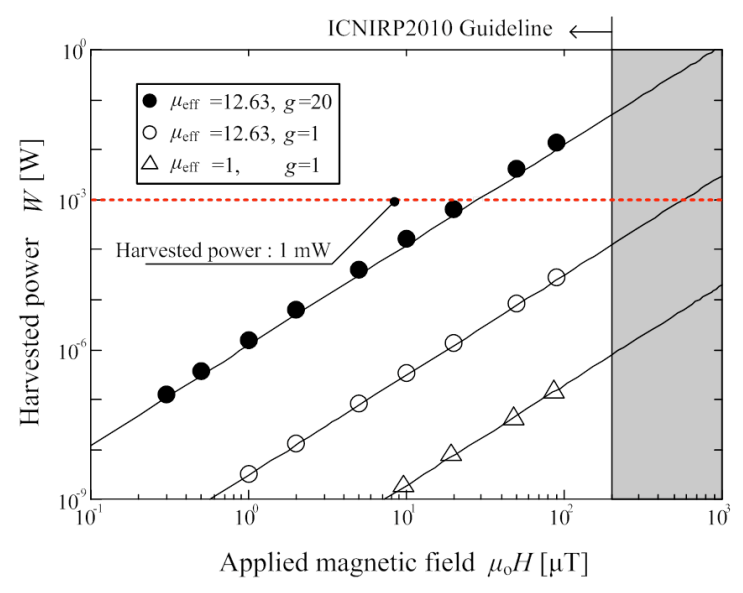

Fig. 6. Harvested power of the module that flange is $20 \mathrm{~mm}$ $(\mathrm{g}=1,20)$ and coil core for alminum $\left(\mu_{\mathrm{eff}}=1, g=1\right)$

\subsection{Two magnetic concentration methods}

Fig. 6 shows an experimental result of harvested power as a function of applied magnetic field. The plots represent measured values, and the lines represent the estimated value from equation (4). In this experiment, we used the module with $45 \mathrm{~mm}$ in length of the magnetic core. The measured values were in good agreement with the estimated values. When we placed this module inside the magnetic flux concentration coil, the harvested energy was dramatically increased. The maximum harvested energy was $13.8 \mathrm{~mW}$ when the applied magnetic field was $90 \mu \mathrm{T}$ at $60 \mathrm{~Hz}$. If we can use a uniform magnetic field of $200 \mu \mathrm{T}$ at $60 \mathrm{~Hz}$, this module can harvest $57 \mathrm{~mW}$ from the applied magnetic field.

\section{Conclusion}

(1) We have introduced energy enhance ratio $\alpha$ which is proportional to $\left(g \times \mu_{\text {eff }}\right)^{2}$. Where $g$ represents the magnetic flux concentration ratio with a magnetic flux concentration coil, $\mu_{\text {eff }}$ represents effective permeability defined by the magnetic flux concentration ratio with a magnetic material.

(2) In this paper, we defined reusable magnetic field of $60 \mathrm{~Hz}$ and the diameter of the harvesting module was $200 \mu \mathrm{T}$ and $20 \mathrm{~mm}$, respectively. The required energy enhance ratio should be larger than 1000 to harvest $1 \mathrm{~mW}$ energy.

(3) A magnetic flux concentration coil consists of an air-core Brooks coil and a resonant capacitor was investigated. From experimental results, we confirmed that this coil can provide an area where the value of $g$ was larger than 20 .

(4) Dumbbell-shaped magnetic cores for the harvesting module were developed. When the length of the core was $45 \mathrm{~mm}$, the energy enhance ratio was 160 which corresponds to the effective permeability of 12.63 .

(5) Incorporating the two magnetic flux concentration methods, we have successfully demonstrated magnetic energy harvesting of $1 \mathrm{~mW}$ with this small module. Our achieved energy enhance ratio was as high as 57,760 . If we can reuse magnetic field of $200 \mu \mathrm{T}$ at $60 \mathrm{~Hz}$, the corresponding energy becomes $57 \mathrm{~mW}$.

\section{References}

1. K. Tashiro, H. Wakiwaka, S. Inoue, Y. Uchiyama, IEEE Trans. Magn., 47, pp. 4441-4444 (2011)

2. K. Tashiro, H. Wakiwaka, Y. Uchiyama, Mater. Sci. Forum, 721, pp. 191-198 (2012)

3. K. Tashiro, H. Wakiwaka, Y. Uchiyama, J. Jpn. Soc. Appl. Electromagn. Mech., 20, pp. 440-445 (2012)

4. ICNIRP, Health phys., 99, pp.818-836 (2010)

5. S. Roundy, P. K. Wright, J. Rabaey, Comput. Commun., 26, pp. 1131-1144 (2003)

6. R. J. M. Vullers, R. V. Schaijk, I. Doms, C. V. Hoof, R. Mertens, Solid-State Electron., vol. 53, pp. 684693 (2009)

7. F. W. Grover, Inductance Calculations, pp.91-105 (2004)

8. K. Tashiro, A. Matsuoka, H. Wakiwaka, Mater. Sci. Forum, 670, pp.275-283 (2011)

9. E. Paperno, A. Grosz, J. Appl. Phys., 105, $07 \mathrm{E} 708$ (2009) 